\title{
Brain Plasticity and Behavior
}

\author{
Bryan Kolb, ${ }^{1}$ Robbin Gibb, and Terry E. Robinson
}

Canadian Centre for Behavioural Neuroscience, University of Lethbridge, Lethbridge, Alberta, Canada (B.K., RG.), and Department of Psychology, University of Michigan, Ann Arbor, Michigan (T.E.R.)

\begin{abstract}
Although the brain was once seen as a rather static organ, it is now clear that the organization of brain circuitry is constantly changing as a function of experience. These changes are referred to as brain plasticity, and they are associated with functional changes that include phenomena such as memory, addiction, and recovery of function. Recent research has shown that brain plasticity and behavior can be influenced by a myriad of factors, including both pre- and postnatal experience, drugs, hormones, maturation, aging, diet, disease, and stress. Understanding how these factors influence brain organization and function is important not only for understanding both normal and abnormal behavior, but also for designing treatments for behavioral and psychological disorders ranging from addiction to stroke.

\section{Keywords addiction; recovery; experi- ence; brain plasticity}

One of the most intriguing questions in behavioral neuroscience concerns the manner in which the nervous system can modify its organization and ultimately its function throughout an individual's lifetime, a property that is often referred to as plasticity. The capacity to change is a fundamental characteristic of nervous systems and can be seen in even the simplest of or- ganisms, such as the tiny worm $C$. elegans, whose nervous system has only 302 cells. When the nervous system changes, there is often a correlated change in behavior or psychological function. This behavioral change is known by names such as learning, memory, addiction, maturation, and recovery. Thus, for example, when people learn new motor skills, such as in playing a musical instrument, there are plastic changes in the structure of cells in the nervous system that underlie the motor skills. If the plastic changes are somehow prevented from occurring, the motor learning does not occur. Although psychologists have assumed that the nervous system is especially sensitive to experience during development, it is only recently that they have begun to appreciate the potential for plastic changes in the adult brain. Understanding brain plasticity is obviously of considerable interest both because it provides a window to understanding the development of the brain and behavior and because it allows insight into the causes of normal and abnormal behavior.

\section{THE NATURE OF BRAIN PLASTICITY}

The underlying assumption of studies of brain and behavioral plasticity is that if behavior changes, there must be some change in organization or properties of the neural circuitry that produces the behavior. Conversely, if neural networks are changed by experience, there must be some corresponding change in the functions mediated by those networks. For the investigator interested in understanding the factors that can change brain circuits, and ultimately behavior, a major challenge is to find and to quantify the changes. In principle, plastic changes in neuronal circuits are likely to reflect either modifications of existing circuits or the generation of new circuits. But how can researchers measure changes in neural circuitry? Because neural networks are composed of individual neurons, each of which connects with a subset of other neurons to form interconnected networks, the logical place to look for plastic changes is at the junctions between neurons, that is, at synapses. However, it is a daunting task to determine if synapses have been added or lost in a particular region, given that the human brain has something like 100 billion neurons and each neuron makes on average several thousand synapses. It is clearly impractical to scan the brain looking for altered synapses, so a small subset must be identified and examined in detail. But which synapses should be studied? Given that neuroscientists have a pretty good idea of what regions of the brain are involved in particular behaviors, they can narrow their search to the likely areas, but are still left with an extraordinarily complex system to examine. There is, however, a procedure that makes the job easier.

In the late 1800s, Camillo Golgi invented a technique for staining a random subset of neurons $(1-5 \%)$ so that the cell bodies and the dendritic trees of individual cells can be visualized (Fig. 1). The dendrites of a cell function as the scaffolding for synapses, much as tree branches provide a location for leaves to grow and be exposed to sunlight. The usefulness of Golgi's technique can be understood by pursuing this arboreal metaphor. There are a number of ways one 


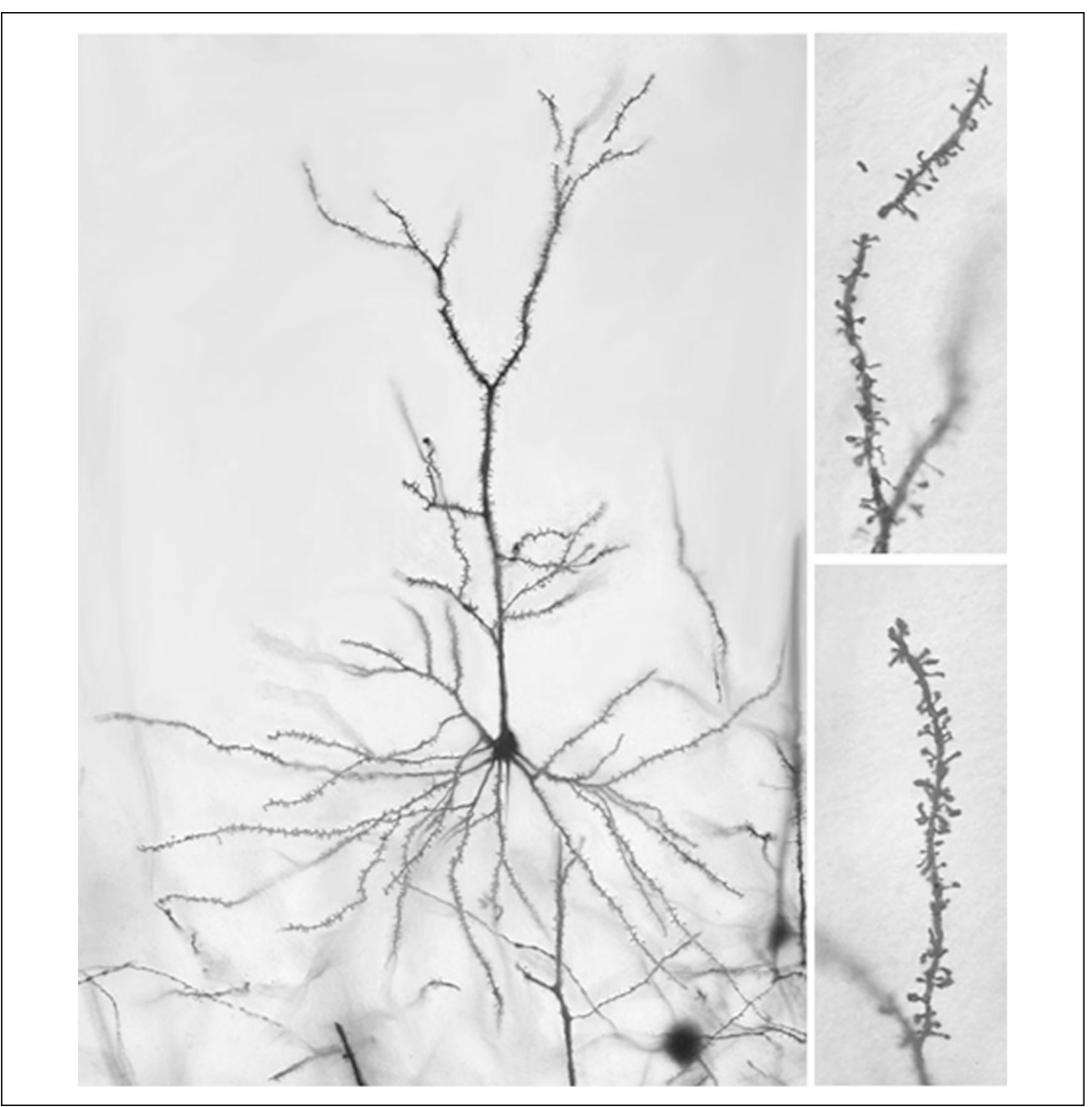

Fig. 1. Photograph of a neuron. In the view on the left, the dendritic field with the extensive dendritic network is visible. On the right are higher-power views of dendritic branches showing the spines, where most synapses are located. If there is an increase in dendritic length, spine density, or both, there are presumed to be more synapses in the neuron.

could estimate how many leaves are on a tree without counting every leaf. Thus, one could measure the total length of the tree's branches as well as the density of the leaves on a representative branch. Then, by simply multiplying branch length by leaf density, one could estimate total leafage. A similar procedure is used to estimate synapse number. About $95 \%$ of a cell's synapses are on its dendrites (the neuron's branches). Furthermore, there is a roughly linear relationship between the space available for synapses (dendritic surface) and the number of synapses, so researchers can presume that increases or decreases in dendritic surface reflect changes in synaptic organization.

\section{FACTORS AFFECTING BRAIN PLASTICITY}

By using Golgi-staining procedures, various investigators have shown that housing animals in complex versus simple environments produces widespread differences in the number of synapses in specific brain regions. In general, such experiments show that particular experiences embellish circuitry, whereas the absence of those experiences fails to do so (e.g., Greenough \& Chang, 1989). Until recently, the impact of these neuropsychological experiments was surprisingly limited, in part because the environmental treat- ments were perceived as extreme and thus not characteristic of events experienced by the normal brain. It has become clear, however, not only that synaptic organization is changed by experience, but also that the scope of factors that can do this is much more extensive than anyone had anticipated. Factors that are now known to affect neuronal structure and behavior include the following:

- experience (both leading preand post-natal)

- psychoactive drugs (e.g., amphetamine, morphine)

- gonadal hormones (e.g., estrogen, testosterone)

- anti-inflammatory agents (e.g., COX-2 inhibitors)

- growth factors (e.g., nerve growth factor)

- dietary factors (e.g., vitamin and mineral supplements)

- genetic factors (e.g., strain differences, genetically modified mice)

- disease (e.g., Parkinson's disease, schizophrenia, epilepsy, stroke)

- stress

- brain injury and leading disease

We discuss two examples to illustrate.

\section{Early Experience}

It is generally assumed that experiences early in life have different effects on behavior than similar experiences later in life. The reason for this difference is not understood, however. To investigate this question, we placed animals in complex environments either as juveniles, in adulthood, or in senescence (Kolb, Gibb, \& Gorny, 2003). It was our expectation that there would be quantitative differences in the effects of experience on synaptic organization, but to our surprise, we also found qualitative differences. Thus, like many investigators before us, we found that the length of dendrites and the density of syn- 
apses were increased in neurons in the motor and sensory cortical regions in adult and aged animals housed in a complex environment (relative to a standard lab cage). In contrast, animals placed in the same environment as juveniles showed an increase in dendritic length but a decrease in spine density. In other words, the same environmental manipulation had qualitatively different effects on the organization of neuronal circuitry in juveniles than in adults.

To pursue this finding, we later gave infant animals 45 min of daily tactile stimulation with a little paintbrush (15 min three times per day) for the first 3 weeks of life. Our behavioral studies showed that this seemingly benign early experience enhanced motor and cognitive skills in adulthood. The anatomical studies showed, in addition, that in these animals there was a decrease in spine density but no change in dendritic length in cortical neurons-yet another pattern of experience-dependent neuronal change. (Parallel studies have shown other changes, too, including neurochemical changes, but these are beyond the current discussion.) Armed with these findings, we then asked whether prenatal experience might also change the structure of the brain months later in adulthood. Indeed, it does. For example, the offspring of a rat housed in a complex environment during the term of her pregnancy have increased synaptic space on neurons in the cerebral cortex in adulthood. Although we do not know how prenatal experiences alter the brain, it seems likely that some chemical response by the mother, be it hormonal or otherwise, can cross the placental barrier and alter the genetic signals in the developing brain.

Our studies showing that experience can uniquely affect the developing brain led us to wonder if the injured infant brain might be repaired by environmental treat- ments. We were not surprised to find that postinjury experience, such as tactile stroking, could modify both brain plasticity and behavior because we had come to believe that such experiences were powerful modulators of brain development (Kolb, Gibb, \& Gorny, 2000). What was surprising, however, was that prenatal experience, such as housing the pregnant mother in a complex environment, could affect how the brain responded to an injury that it would not receive until after birth. In other words, prenatal experience altered the brain's response to injury later in life. This type of study has profound implications for preemptive treatments of children at risk for a variety of neurological disorders.

\section{Psychoactive Drugs}

Many people who take stimulant drugs like nicotine, amphetamine, or cocaine do so for their potent psychoactive effects. The long-term behavioral consequences of abusing such psychoactive drugs are now well documented, but much less is known about how repeated exposure to these drugs alters the nervous system. One experimental demonstration of a very persistent form of drug experiencedependent plasticity is known as behavioral sensitization. For example, if a rat is given a small dose of amphetamine, it initially will show a small increase in motor activity (e.g., locomotion, rearing). When the rat is given the same dose on subsequent occasions, however, the increase in motor activity increases, or sensitizes, and the animal may remain sensitized for weeks, months, or even years, even if drug treatment is discontinued.

Changes in behavior that occur as a consequence of past experience, and can persist for months or years, like memories, are thought to be due to changes in patterns of synaptic or- ganization. The parallels between drug-induced sensitization and memory led us to ask whether the neurons of animals sensitized to drugs of abuse exhibit long-lasting changes similar to those associated with memory (e.g., Robinson \& Kolb, 1999). A comparison of the effects of amphetamine and saline treatments on the structure of neurons showed that neurons in amphetamine-treated brains had greater dendritic material, as well as more densely organized spines. These plastic changes were not found throughout the brain, however, but rather were localized to regions such as the prefrontal cortex and nucleus accumbens, both of which are thought to play a role in the rewarding properties of these drugs. Later studies have shown that these druginduced changes are found not only when animals are given injections by an experimenter, but also when animals are trained to self-administer drugs, leading us to speculate that similar changes in synaptic organization will be found in human drug addicts.

\section{Other Factors}

All of the factors we listed earlier have effects that are conceptually similar to the two examples that we just discussed. For instance, brain injury disrupts the synaptic organization of the brain, and when there is functional improvement after the injury, there is a correlated reorganization of neural circuits (e.g., Kolb, 1995). But not all factors act the same way across the brain. For instance, estrogen stimulates synapse formation in some structures but reduces synapse number in other structures (e.g., Kolb, Forgie, Gibb, Gorny, \& Rowntree, 1998), a pattern of change that can also be seen with some psychoactive drugs, such as morphine. In sum, it now appears that virtually any manipulation 
that produces an enduring change in behavior leaves an anatomical footprint in the brain.

\section{CONCLUSIONS AND ISSUES}

There are several conclusions to draw from our studies. First, experience alters the brain, and it does so in an age-related manner. Second, both pre- and postnatal experience have such effects, and these effects are long-lasting and can influence not only brain structure but also adult behavior. Third, seemingly similar experiences can alter neuronal circuits in different ways, although each of the alterations is manifest in behavioral change. Fourth, a variety of behavioral conditions, ranging from addiction to neurological and psychiatric disorders, are correlated with localized changes in neural circuits. Finally, therapies that are intended to alter behavior, such as treatment for addiction, stroke, or schizophrenia, are likely to be most effective if they are able to further reorganize relevant brain circuitry. Furthermore, studies of neuronal structure provide a simple method of screening for treatments that are likely to be effective in treating disorders such as dementia. Indeed, our studies show that the new generation of antiarthritic drugs (known as COX-2 inhibitors), which act to reduce inflammation, can reverse agerelated synaptic loss and thus ought to be considered as useful treatments for age-related cognitive loss.

Although much is now known about brain plasticity and behavior, many theoretical issues remain. Knowing that a wide variety of experiences and agents can alter synaptic organization and behavior is important, but leads to a new question: How does this happen? This is not an easy question to answer, and it is certain that there is more than one an- swer. We provide a single example to illustrate.

Neurotrophic factors are a class of chemicals that are known to affect synaptic organization. An example is fibroblast growth factor-2 (FGF-2). The production of FGF-2 is increased by various experiences, such as complex housing and tactile stroking, as well as by drugs such as amphetamine. Thus, it is possible that experience stimulates the production of FGF-2 and this, in turn, increases synapse production. But again, the question is how. One hypothesis is that FGF-2 somehow alters the way different genes are expressed by specific neurons and this, in turn, affects the way synapses are generated or lost. In other words, factors that alter behavior, including experience, can do so by altering gene expression, a result that renders the traditional gene-versus-environment discussions meaningless.

Other issues revolve around the limits and permanence of plastic changes. After all, people encounter and learn new information daily. Is there some limit to how much cells can change? It seems unlikely that cells could continue to enlarge and add synapses indefinitely, but what controls this? We saw in our studies of experience-dependent changes in infants, juveniles, and adults that experience both adds and prunes synapses, but what are the rules governing when one or the other might occur? This question leads to another, which is whether plastic changes in response to different experiences might interact. For example, does exposure to a drug like nicotine affect how the brain changes in learning a motor skill like playing the piano? Consider, too, the issue of the permanence of plastic changes. If a person stops smoking, how long do the nicotine-induced plastic changes persist, and do they affect later changes?

One additional issue surrounds the role of plastic changes in disordered behavior. Thus, although most studies of plasticity imply that remodeling neural circuitry is a good thing, it is reasonable to wonder if plastic changes might also be the basis of pathological behavior. Less is known about this possibility, but it does seem likely. For example, drug addicts often show cognitive deficits, and it seems reasonable to propose that at least some of these deficits could arise from abnormal circuitry, especially in the frontal lobe.

In sum, the structure of the brain is constantly changing in response to an unexpectedly wide range of experiential factors. Understanding how the brain changes and the rules governing these changes is important not only for understanding both normal and abnormal behavior, but also for designing treatments for behavioral and psychological disorders ranging from addiction to stroke.

\section{Recommended Reading}

Kolb, B., \& Whishaw, I.Q. (1998). Brain plasticity and behavior. Annual Review of Psychology, 49, 43-64.

Robinson, T.E., \& Berridge, K.C. (in press). Addiction. Annual Review of Psychology.

Shaw, C.A., \& McEachern, J.C. (2001). Toward a theory of neuroplasticity. New York: Taylor and Francis.

Acknowledgments-This research was supported by a Natural Sciences and Engineering Research Council grant to B.K. and a National Institute on Drug Abuse grant to T.E.R.

\section{Note}

1. Address correspondence to Bryan Kolb, CCBN, University of Lethbridge, Lethbridge, AB, Canada T1K 3M4.

\section{References}

Greenough, W.T., \& Chang, F.F. (1989). Plasticity of synapse structure and pattern in the cerebral cortex. In A. Peters \& E.G. Jones (Eds.), Cere- 
bral cortex: Vol. 7 (pp. 391-440). New York: Plenum Press.

Kolb, B. (1995). Brain plasticity and behavior. Mahwah, NJ: Erlbaum.

Kolb, B., Forgie, M., Gibb, R., Gorny, G., \& Rowntree, S. (1998). Age, experience, and the changing brain. Neuroscience and Biobehavioral Reviews, $22,143-159$
Kolb, B., Gibb, R., \& Gorny, G. (2000). Cortical plasticity and the development of behavior after early frontal cortical injury. Developmental Neuropsychology, 18, 423-444.

Kolb, B., Gibb, R., \& Gorny, G. (2003). Experience-dependent changes in dendritic arbor and spine density in neocortex vary with age and sex. Neurobiology of Learning and Memory, $79,1-10$.

Robinson, T.E., \& Kolb, B. (1999). Alterations in the morphology of dendrites and dendritic spines in the nucleus accumbens and prefrontal cortex following repeated treatment with amphetamine or cocaine. European Journal of Neuroscience, 11, 1598-1604.

\section{The Malicious Serpent: Snakes as a Prototypical Stimulus for an Evolved Module of Fear}

\section{Arne Öhman ${ }^{1}$ and Susan Mineka}

Department of Clinical Neuroscience, Karolinska Institute, Stockholm, Sweden (A.Ö.), and Department of Psychology, Northwestern University, Evanston, Illinois (S.M.)

\section{Abstract}

As reptiles, snakes may have signified deadly threats in the environment of early mammals. We review findings suggesting that snakes remain special stimuli for humans. Intense snake fear is prevalent in both humans and other primates. Humans and monkeys learn snake fear more easily than fear of most other stimuli through direct or vicarious conditioning. Neither the elicitation nor the conditioning of snake fear in humans requires that snakes be consciously perceived; rather, both processes can occur with masked stimuli. Humans tend to perceive illusory correlations between snakes and aversive stimuli, and their attention is automatically captured by snakes in complex visual displays. Together, these and other findings delineate an evolved fear module in the brain. This module is selectively and automatically activated by once-threatening stimuli, is relatively encapsulated from cognition, and derives from specialized neural circuitry.

\section{Keywords evolution; snake fear; fear module}

Snakes are commonly regarded as slimy, slithering creatures worthy of fear and disgust. If one were to believe the Book of Genesis, humans' dislike for snakes resulted from a divine intervention: To avenge the snake's luring of Eve to taste the fruit of knowledge, God instituted eternal enmity between their descendants. Alternatively, the human dislike of snakes and the common appearances of reptiles as the embodiment of evil in myths and art might reflect an evolutionary heritage. Indeed, Sagan (1977) speculated that human fear of snakes and other reptiles may be a distant effect of the conditions under which early mammals evolved. In the world they inhabited, the animal kingdom was dominated by awesome reptiles, the dinosaurs, and so a prerequisite for early mammals to deliver genes to future generations was to avoid getting caught in the fangs of Tyrannosaurus rex and its relatives. Thus, fear and respect for reptiles is a likely core mammalian heritage. From this perspective, snakes and other rep- tiles may continue to have a special psychological significance even for humans, and considerable evidence suggests this is indeed true. Furthermore, the pattern of findings appears consistent with the evolutionary premise.

\section{THE PREVALENCE OF SNAKE FEARS IN PRIMATES}

Snakes are obviously fearsome creatures to many humans. Agras, Sylvester, and Oliveau (1969) interviewed a sample of New Englanders about fears, and found snakes to be clearly the most prevalent object of intense fear, reported by $38 \%$ of females and $12 \%$ of males.

Fear of snakes is also common among other primates. According to an exhaustive review of field data (King, 1997), 11 genera of primates showed fear-related responses (alarm calls, avoidance, mobbing) in virtually all instances in which they were observed confronting large snakes. For studies of captive primates, King did not find consistent evidence of snake fear. However, in direct comparisons, rhesus (and squirrel) monkeys reared in the wild were far more likely than labreared monkeys to show strong phobiclike fear responses to snakes (e.g., Mineka, Keir, \& Price, 1980). That this fear is adaptive in the wild is further supported by independent field reports of large snakes attacking primates (M. Cook \& Mineka, 1991).

This high prevalence of snake fear in humans as well as in our 